\title{
Investing Data with Untrusted Parties using HE
}

\author{
Mark Dockendorf, Ram Dantu, Kirill Morozov and Sanjukta Bhowmick \\ Department of Computer Science, University of North Texas, Denton, U.S.A.
}

Keywords: Homomorphic, Encryption, Graphs, Privacy.

\begin{abstract}
Data such as an individual's income, favorite sports team, typical commute route, vehicle maintenance history, medical records, etc. are typically not useful for making large-scale decisions such as where to build a new hospital, identifying which roads are in need of upkeep, and the like. However, aggregates of of these data across hundreds of individuals are useful to governments and to companies. Data cooperatives/unions offer a place for individuals to store their data and a service of data aggregation and interpretation to governments, non-profit organizations, and businesses while maintaining individuals' anonymity. We propose the use of anonymization techniques coupled with graph algorithms over homomorphically encrypted (HE) graphs as a basis of analysis for this accumulated data. We believe this approach ensures individuals' privacy and anonymity while preserving the usefulness of the plaintext data.
\end{abstract}

\section{INTRODUCTION AND MOTIVATION}

Data has become one of the world's most prized commodities. Its value lies in its potential to provide insight, be it to corporations, interest groups, or governments (World Economic Forum, 2011). Large amounts of data are harvested by these various entities for any number of economic, social, or cultural purposes. Purchase history, browsing history, medical records, financial information-there is no limit to the value of large datasets, and some organization somewhere will have a use for them.

But who is in control of the world's data? As of now, data is largely the property of a few influential companies (Walsh, 2019). This raises several concerns. Firstly, individuals are not in control of their data. They do not know who has access to their data and how they are using it. Secondly, there is no guarantee that the data is secure and properly anonymized. Identifiable information may be in plain view to those who do not need to see it.

The formation of data cooperatives addresses both concerns. A data cooperative is a legal construct wherein members voluntarily pool data for the benefit of the group (Geuns et al., 2020). Through a data cooperative, individuals are given the choice to control their data. They can choose to share their data, who can use it, and how it is used. Furthermore, co-ops have a vested interest in keeping their members safe against data theft and advocating for their members' rights (Ligett et al., 2019). This construct supports the privacy and security of the individual's information.

Despite being a somewhat novel concept, numerous data cooperatives have already formed successfully. For example, there exists a Swiss co-op, Healthbank, that aggregates personal health data for the purpose of research and medical advancement. Another project called Driver's Seat is a co-op comprised of on-demand drivers (e.g., Uber or Lyft drivers) who choose to share data related to their travels. This allows the members of the co-op to optimize their workflow using insights from the data pool. More recently, there is interest in creating a data cooperative that collects information about contact tracing such that super-spreaders of COVID-19 can be identified (Dockendorf et al., 2021). Authors have also used data cooperatives to mine and chain fake data. This work was selected as one of the hard problems in the science of security and we will be presenting at the Hot Topics in Science of Security conference (Salau et al., 2021; Salau et al., 2021). These applications demonstrate the wide variety of functions that a data cooperative can serve.

Data cooperatives are useful, but how can a coop ensure that their data is secure? A key concern is that information from a cooperative can be misused to obtain confidential data about individuals. Pieces of information that aren't anonymized properly can be cross-referenced to obtain an individual's entire pro- 
file, including emails, addresses, and social security information. This personally identifiable information can be used for identity theft, blackmail, or surveillance.

These security concerns can be mitigated through graph anonymization and homomorphic encryption. All data, particularly that from computer logs, network logs, and financial logs, can be represented by graphs. These graphs can be anonymized by modifying their structure using k-core decomposition. This method involves edge swapping at lower cores such that the structure of the higher cores is retained, preserving the network's structure while obscuring identifiable information. These graphs can be secured further using homomorphic encryption, a cornerstone of modern cryptographic protocol design. To securely analyze this encrypted data, graph operations are layered over the homomorphic encryption. By using these two methods, a multi-modal security framework is built that allows users of the data cooperative to see data insights without accessing the data itself.

\section{BACKGROUND}

Network Analysis. Complex systems of interacting entities such as social networks, computer networks, and financial systems are naturally represented as graphs. In this context, network analysis algorithms form a basis for secure data analytics in the areas such as electronic commerce and computational epidemiology. Network analysis is an active area of research, where graph algorithms are used to explore properties of complex interactive systems. Of particular importance are centrality metrics that are used for measuring the importance of entities represented as vertices. For example, in a contact graph, vertices with high centrality point at potential superspreaders. Some of the popular metrics are degree centrality (vertex degree), closeness centrality (mean distance of the vertex from other vertices), betweenness centrality (fraction of shortest paths passing through the vertex), and eigenvector centrality and its variations such as PageRank (the number of important neighbors of the vertex) (Newman, 2010).

Homomorphic Encryption (HE). Such encryption schemes support computation on encrypted data, without access to a private key. Of particular interest in this context are the so called fully homomorphic encryption (FHE) schemes, which support computation of arbitrary functions-see (Halevi, 2017) for a comprehensive survey. These cryptosystems ideally fit the scenario of data cooperatives since the former provide confidentiality of members' personal data while enabling computations on them. Multi-key FHE schemes introduced by Lopez-Alt et al. (LópezAlt et al., 2012) will be particularly useful in this setting, as they allow computation on data encrypted using different keys. FHE schemes are known to be demanding in terms of computational resources. Therefore, the main challenge for their deployment in the practical scenario is to ensure that a wide range of algorithms can be run on large amounts of encrypted data.

\section{PROPOSED ARCHITECTURE}

Figure 1 shows a high-level block diagram of of how a data union/cooperative that employs HE graphs for data analysis might be structured. Importantly, this diagram is for a data union that does not utilize cloud services.

Individuals or apps (on behalf of individuals) submit data to the data union through the Personal Data API. These individuals own their respective data. Multiparty encryption is used on all data submitted, and all data owners are assumed to be opt-out of all data analysis unless they grant consent to either a category of usage or consent to participate in a specific data study. This API validates the data to ensure it meets a standard of input and enables the multiparty encryption for permanent storage. The data owner may retrieve this information from the data union by validating their credentials (ie. with username and password). However, the data returned will only be decrypted by the data union, so the data owner must still possess their own key to fully unlock it. When a data owner grants new usage permission on their data, the Personal Data API will send their data to them (with both data union and data owner encryption inplace). The data owner will unlock the data using his or her key, and transmit back to the data union. Importantly, not all data need be transmitted every time, only the relevant data will be transmitted for decryption.

Encrypted Storage keeps a copy of all input data in encrypted form using multiparty encryption. The individual will keep a copy of their key, which could be a physical USB keyring or similar, and the data union/cooperative keeps the other key. If the data union/cooperative wants to use a set of personal information to generate aggregates, consent (and the key) of the individual is required. The storage used should be resilient without presence of electricity (ie. optical storage). This portion of the data union/cooperative is modeled after a traditional bank's safety deposit boxes where (i) identity verification, (ii) the owner's 


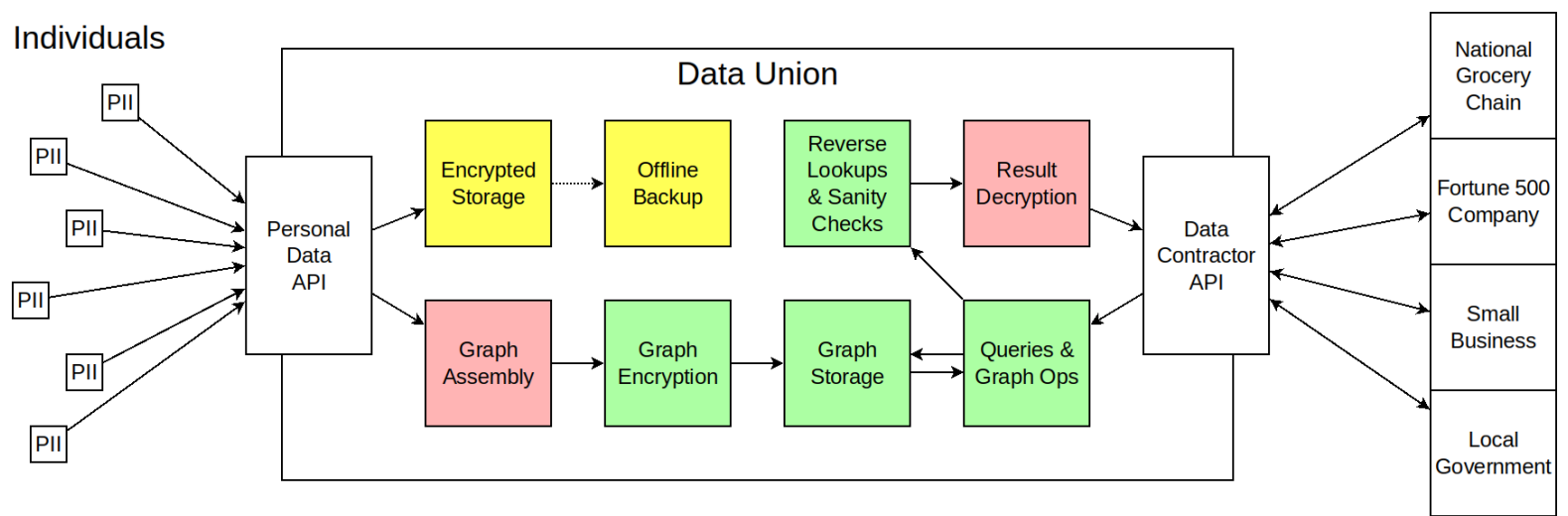

Figure 1: High-level diagram for a data union. Architecture modules that are red handle sensitive data and either (i) handle plaintext sensitive data or (ii) may possess the key to the sensitive data they handle. Modules in green handle exclusively HE graph data and public graph data. The yellow blocks, use traditional multiparty encryption, so the data stored is not computable.

key, and (iii) the bank's key are all required for access. The data union should also maintain an offline backup of this encrypted storage that is updated periodically (ie. daily, weekly, etc).

Graph Assembly takes data (currently not encrypted or with the infrastructure processing it knowing the key) and assembles graphs based on desired relations. Each individual's data can be encrypted independent of one-another: this allows the data union to access the data with the owner's consent, transform the data into the appropriate form, and immediately forward the organized data to Graph Encryption and forget the plaintext values. Each data point can be used in the creation of multiple graphs and matrices that transform data, mapping from one type of graph to another (ie. people to roads used in their commutes), will also be created. This subsystem shreds plaintext data (overwrites with random data) once the values have been encrypted.

Graph Encryption takes values created by Graph Assembly and converts them to HE Graphs; notably, individual columns/rows can be encrypted without all others being present. This allows the plaintext values to be removed from system memory. For each attribute vector, there is an associated set of encryption schemes to encrypt the data under. A graph or attribute vector may be encrypted in many forms (ie binary, fixed-point, integer). This subsystem is responsible for updating HE graphs in Graph Storage as their values change. This subsystem shreds plaintext data once it is no longer needed and forgets ciphertext data once it is stored in Graph Storage.

Graph Storage keeps HE Graphs and answers queries. Functionally, this subsystem is a database. This subsystem will deny read access to ciphertext graphs for all subsystems other than Graph Opera- tions.

Graph Operations performs operations requested by the Data Contractor API after basic validation. Specifically, this subsystem (1) parses aggregate algebra into operations (a series of parallel operations) and (2) executes the requested matrix math. This subsystem has read access to graph storage and is responsible for performing HE Graph operations (centralities, BFSs, etc) and data transformations.

Reverse Lookups \& Sanity Checking ensures no one's personal information will be exposed because of the query (ie. enough participants to be sufficiently anonymous). This subsystem is also responsible for performing lookups that transform indices back into the appropriate names (ie. road segment index 51253 to "5500-6000 block of Main St."). Naturally, this module does not have a lookup table for data like people's names: this would violate their privacy.

Result Decryption decrypts the aggregated results, which should not be able to identify any individual. Companies, governments, and non-profit organizations submit queries to the Data Contractor API and receive their results when this module finishes.

The Data Contractor API accepts, validates, and answers requests for aggregates by companies, governments, etc. This API will return an error when a referenced value is not in a lookup table or when the starting value for an aggregate is not part of a public data set. These error might happen when an aggregate references something that is not public data, such as a person's name, or the aggregate starts with a crafted vector on vertices that represents data that is not public.

Architecture Enabling Multi-modal Privacy. Table 1 shows a comparison of the two methods ensuring data privacy: anonymization and HE. In order to 
capitalize on both of these methods, we propose that encrypted graphs be placed on the cloud, which we conditionally call "public"-it will be accessible by entities in the data co-op who need access to specific information on the user data. The anonymized data will be placed on the cloud, which we will call "private". This cloud will provide anonymized graphs to those in the co-op who need to see the entire network structure (e.g., for statistical analysis), but who do not need specific user identities.

\section{APPLICATIONS FOR THE PROPOSED METHODS}

Table 1: Comparison of graph anonymization and graph encryption approaches.

\begin{tabular}{|l|l|l|}
\hline & Graph Anonymization & Graph Encryption \\
\hline Function & $\begin{array}{l}\text { Obscuring: (i) links be- } \\
\text { tween vertices and in- } \\
\text { dividuals (identity), (ii) } \\
\text { links between pairs of } \\
\text { individuals, and (iii) } \\
\text { inference of attributes } \\
\text { among individuals. }\end{array}$ & $\begin{array}{l}\text { Data confiden- } \\
\text { tiality, and hence } \\
\text { data leakage, e.g., } \\
\text { due to unautho- } \\
\text { rized access. }\end{array}$ \\
\hline Security & $\begin{array}{l}\text { Data are available but } \\
\text { distorted to obscure } \\
\text { sensitive information. }\end{array}$ & $\begin{array}{l}\text { Data are sealed } \\
\text { and hence their } \\
\text { privacy is pre- } \\
\text { served. }\end{array}$ \\
\hline $\begin{array}{l}\text { Inter- } \\
\text { operability }\end{array}$ & $\begin{array}{l}\text { GDPR: Cross-border } \\
\text { data transfers (between } \\
\text { countries) mandate } \\
\text { anonymization }\end{array}$ & $\begin{array}{l}\text { Encryption not al- } \\
\text { lowed for cross- } \\
\text { border data trans- } \\
\text { fers. }\end{array}$ \\
\hline
\end{tabular}

Ensuring Privacy Through Anonymization and Homomorphic Encryption. The two main methods of protecting privacy and security of data are (i) Anonymization and (ii) Encryption. As per their different features, as listed in Table 1, we posit that data analysts who need to explore new algorithms work with the anonymized graph, and co-op members who need data insights use the encrypted graph. Hence, our framework includes homomorphic encryption for processing queries on the encrypted networks, and anonymization based on core-periphery structure of the graph for analyzing structural properties of the networks.

Graph Anonymization Using K-core Decomposition. The primary issue of graph anonymization is that the anonymized graph should retain the important structural properties of the network, while obscuring information that can identify an individual to a node in the network. We posit that anonymizing graphs based on their k-core property is particularly suited for our application because of the following proper- ties:

1. Utility. It has been demonstrated (Meyer et al., 2014; Laishram et al., 2018) that high centrality vertices have higher core numbers. Moreover, kcore is used as a submodule to compute clusters and fast dissemination algorithms which are based on super-spreaders.

2. Accuracy. All anonymization techniques change the structure of the network, which can affect accuracy of the analysis. We have shown (Sakar et al., 2018) that changes are more disruptive within the inner cores of the network, than in the peripheral cores. Therefore, retaining structure of the inner cores will reduce the effect of noise.

3. Efficiency. Compared to most other anonymization methods, computing k-core of graphs is much faster with complexity of only $O\left(d_{\max }|V|+|E|\right)$. We aim to develop anonymization algorithms of comparable complexity.

Most graph anonymization algorithms try to minimize the number of changes. Our studies in (Ufimtsev et al., 2016; Cheon et al., 2018) have shown that the changes to the higher cores disrupt the centrality values more than changes to the periphery.

We therefore propose to develop an anonymization scheme that disrupts the periphery but retains the structure at the higher cores. We achieve this using the two steps: (i) edge swapping for vertices with lower cores; and (ii) adding nodes and edges to preserve $k$ (core, property) anonymity at higher cores.

Edge Swapping at Lower Cores. Swapping edges between nodes of low core numbers, i.e., nodes at the periphery, retain the overall degree distribution of the graph, but changes the degrees of the individual nodes whose edges were swapped. This leads to a restructuring of the periphery of the graph (see Figure 2(b)). Rationale. This step helps in reducing active attacks to de-anonymize the network. Active attacks (Narayanan et al., 2009) are done by planting new entities (nodes) to the original network before it gets anonymized and linking these entities to some important nodes. Once the network is anonymized, the attacker knows the structure of the nodes that he planted and can identify them in the anonymized graph. He can then use links in these nodes to identify important nodes.

Since the planted entities rarely occupy an important position in the graph, they are likely to have lower core numbers. Edge swapping is likely to distort the structure of the planted nodes (and other peripheral nodes), making it difficult for the attacker to identify them after anonymization. For example, let the attacker create a planted subgraph of a chain of degree- 


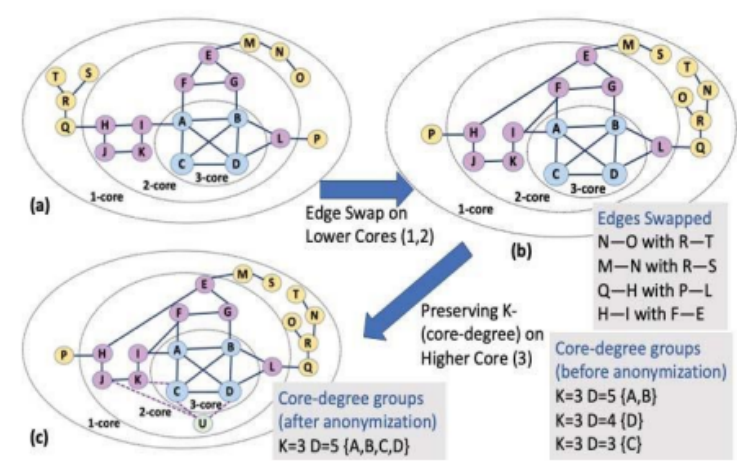

Figure 2: $K$-core decomposition-based graph anonymization. (a) Original graph. (b) Graph after edge swapping. The periphery structure (cores 1,2) is disrupted. (c) Graph after $k$-(core, degree) anonymity. Vertices in the higher core (3), all have the same degree and core number.

1 core- 1 node connected to a degree- 3 core- 2 node (P-L in Figure 2(a)), which is connected to high core nodes. After edge swapping disrupts the periphery, the attacker cannot find his planted subgraph.

Preserving $\boldsymbol{k}$-(core, property) Anonymity at Higher Cores. The $k$-(core, property) anonymity asserts that for a given vertex property, there would be at least $\mathrm{k}$ vertices with high core numbers that have the same core number and the same value of the property. For example, if the property is degree, then there should be at least $\mathrm{k}-1$ vertices with the same core number and the same degree (see Figure 2(c)). We aim to modify the graph by adding nodes to the lower cores and connecting them with higher cores, as that will reduce the disruption of the network.

Rationale. Consider that the attacker knows some vertices with a unique property. The anonymization process aims to create $k$ vertices with that property, such that the attacker can guess the vertices correctly with probability of only $1 / k$.

Similar schemes have been proposed to obtain a set of vertices of the same property, such as k-degree anonymity (Chen et al., 2020) (degree), (k,d)-core anonymity (Assam et al., 2014) (core number) and kisomorphism (Cheng et al., 2010) (subgraphs). Our approach of anonymizing based on pairs rather than only one element provides more flexibility of grouping the nodes and modifying the structure of the network. For example, in Figure 2(c) after adding a new vertex $\mathrm{U}$ and connecting $\mathrm{C}$ to $\mathrm{K}$, all the vertices in core 3 have the same core number and the same degree.

Novelty of Our Approach. The novelty of our approach is that we partition the network into smaller subgraphs that contain or primarily contribute to data analysis (the higher cores), and the larger subgraphs that do not have as much effect on the analysis (the lower cores). Since there are fewer nodes with high core numbers, we apply aggressive anonymization techniques, such as k-(core, property) anonymization. For the lower cores, with larger number of vertices, there is "safety in numbers" and we can apply less stringent and thus less expensive techniques.

Homomorphic Encryption Enabling Graph Analysis. We will develop a framework for computing graph algorithms on encrypted data. We will focus on algorithms related to centrality and clustering.

Secure Matrix Multiplication Mechanism. This mechanism will be at the core of our framework. We will depart from the encrypted matrix multiplication algorithm by Jiang et al. (Jiang et al., 2018).

As bootstrapping time typically dominates the cost of computation over encrypted data, it is important to minimize the multiplicative depth of the circuit to be computed. Jiang et al. deploy the fully homomorphic encryption scheme HEAAN by Cheon et al. (Cheon et al., 2017; Cheon et al., 2018) and an intricate combination of ciphertext packing, rotations, and SIMD-like parallelization to run the multiplication of $\mathrm{d} \times \mathrm{d}$ dense matrices using $\mathrm{O}(\mathrm{d})$ homomorphic operations with multiplicative depth one. When analyzing data from the data co-op pool, we wish to make considerations for sparse matrices. Therefore, we will develop our own secure matrix computation mechanism, leveraging parallelization used, e.g., in the sparse matrix multiplication algorithm of Buluc et al. (Bulu c $_{3}$ et al., 2009). We will also use the HEAAN scheme, as we will need matrix computation over the reals, which this scheme is designed to support. Figure 3 presents a high-level overview of matrix operations to be covered by our approach. We next sketch two examples of how this framework will support network analysis.

Secure Computation of Label Propagation. To exemplify our methodology, let us consider our proposed secure computation of clustering in detail. Specifically, we will implement the label propagation algorithm of (Zhu et al., 2003). Typically, label propagation is computed by applying matrix multiplication iteratively. However, this may result in prohibitively high multiplication depth, and hence computational cost. Zhu and Ghahramani (Zhu et al., 2003) presented the following fixed-point solution to the label propagation, which will allow us to avoid iterations (Mallawaarachchi, 2020; Zhukov, 2015).

Let $A$ denote the adjacency matrix of a graph. Let $D$ be a degree matrix, which is a diagonal matrix whose diagonal elements are computed as $d_{i}=\Sigma_{i} a_{i j}$, where $a_{i j}$ are the elements of $A$. For simplicity of this presentation, we will assume that the probability of transitioning from a node its neighbors is the same 


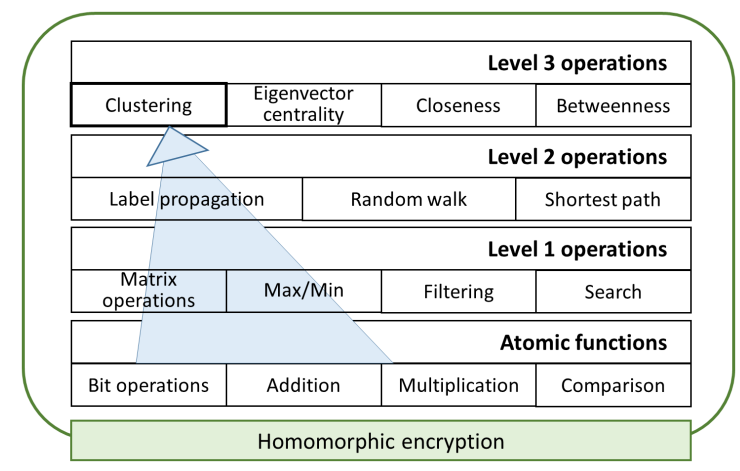

Figure 3: The proposed framework for graph network analysis on encrypted data. The listed graph operations will be implemented to support the data cooperative graph algorithms. We plan to contribute the corresponding libraries to the software projects such as PALISADE. The blue arrow highlights the operations underlying the clustering algorithm.

for each neighbor. In the label propagation algorithm, given an adjacency matrix and labels for the labeled nodes-which we will represent using the matrix $Y_{l^{-}}$ we will compute a vector of labels for the unlabeled nodes. Again, for simplicity, we will assume that the following two steps will be done by the data enabler as preprocessing:

1. Compute the transition probability matrix $T=$ $D^{-1} A$. As a result of this computation, this matrix will have the following form: $T=\left[\begin{array}{cc}I & 0 \\ T_{u l} & T_{u u}\end{array}\right]$, where $I$ is the identity matrix, and $T_{u u}$ and $T_{u l}$ are the submatrices of the probability transition matrix, which are responsible for probability to get from unlabeled nodes to unlabeled nodes, and that to get from unlabeled nodes to labeled nodes, respectively.

2. Compute the matrix $T^{\prime}=\left(I-T_{u u}\right)^{-1} \cdot T_{u l}$, which represented the fixed-point solution.

For simplicity, we assume that the data enabler will send to the cloud a homomorphic encryption of $T^{\prime}$ (denoted by $\operatorname{Enc}\left(T^{\prime}\right)$ ) instead of encryption of the adjacency matrix $A$. Implementation of the above computation steps over an encrypted adjacency matrix is left as a future work. Now, as evidenced in (Zhu et al., 2003; Mallawaarachchi, 2020), computation of the label propagation reduces to $(i)$ a matrix multiplication of $T$ by the label matrix $Y_{l}$, and then (ii) computations of the maximums over the rows of the resulting matrix. Let $n$ be the number of unlabeled nodes, and let $Y_{u}$ denote their label vector (which is to be computed).

\section{EXPERIMENTS}

We successfully implemented breadth-first search, degree centrality, farness centrality (the reciprocal of closeness centrality), and page rank on HE graphs with greater than $99.999 \%$ accuracy for all algorithms when compared to a cleartext calculation. Most of these results are not optimized and are preliminary in nature. Heuristically choosing optimized configurations for the HE schemes that encrypt graphs is part of our active research. These results prove that it is possible to run well-established graph operations on HE graphs with high accuracy.

Algorithm Sketch 1: HE Degree Centrality.

( 1 and 2 may be done offline)

Input: $G$, an adjacency matrix.

Output: $v$

1. Trusted machine selects prime modulus, $p$, such that $p>V(G)$, and initializes BFVrns scheme.

2. Trusted machine encrypts $G$, into column-packed ciphertexts, forming $c G$, which is stored for later use. 3. When degree centrality is invoked, cloud machine(s) sum all columns of $c G$, return cipher vector, $c v$, to trusted machine.

4. Trusted machine decrypts $c v$ into $v$ and returns $v$ to client.

Degree centrality is the fastest of all algorithms currently as it utilizes packed ciphertexts (each column of $G$ is encrypted into a single ciphertext), which allows for vectorization to improve performance. Notably, degree calculation is faster than encryption now: calculation was optimized, encryption is not yet optimized.

\section{Degree Calculation Time vs Graph Size}

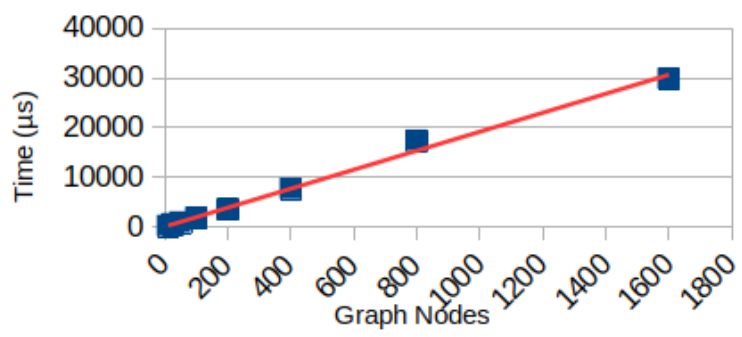

Figure 4: Degree centrality calculation is near-linear time due to packed ciphertexts.

Among other algorithms, we implemented a preliminary binary BFS (binBFS). Unlike degree centrality, which uses somewhat-homomorphic encryption (SHE), this algorithm uses fully-homomorphic encryption (FHE). As a result, this algorithm is slower but can be called any number of times. The core 
of binBFS is a naive binary matrix multiply. This algorithm repeatedly calls $b=B_{G} b$, where $B_{G}=$ $\operatorname{BinFHE}(G)$. This work is preliminary and has not been optimized.

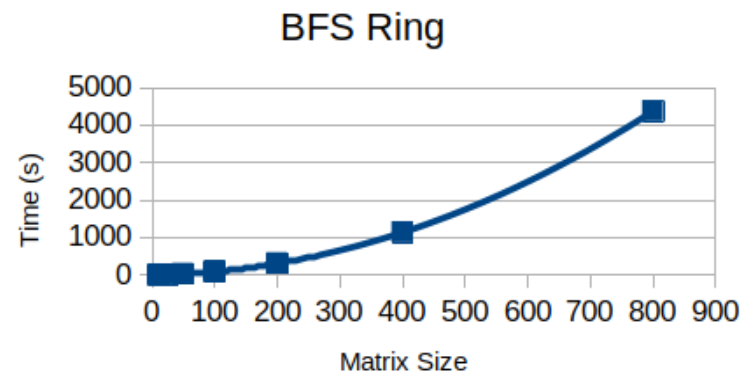

Figure 5: A single ring of expansion on a breadth-first search requires $O\left(n^{2}\right)$ time as a result of the current naive binary matrix-vector multiply.

Farness centrality is another fast algorithm due to packed ciphertexts. The calculation of the ciphertext distance matrix, $D_{G}$, is currently not optimized and very slow. However, calculating $D_{G}$ can be started once $G_{H E}$ is received and is not applicable to transformation matrices (discussed later).

\section{Time to Compute Farness}

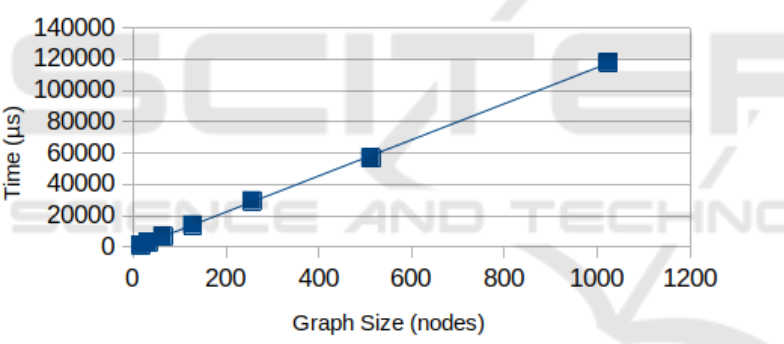

Figure 6: Unlike the calculation of the distance matrix, the time to calculate farness centrality is approximately $O(n)$.

\section{RELATED WORK}

Our work is on HE operations for graphs with the option for use on any generic distributed system without compromising data privacy. This work runs parallel to similar efforts for data analysis such as machine learning over homomorphically encrypted data and MIT's Enigma, which offers data integrity over a distributed system. These analysis could be completed on untrusted distributed systems, such as MIT Enigma(Zyskind et al., 2015), without compromising data privacy. In addition to inter-personal graph constructs, personal data can be assembled into attribute vectors, encrypted, and used for machine learning(Aslett et al., 2015), which, in turn can be part of a federated learning technique. Even when these other schemes are used, our approach offers the ability to refine training datasets based on graph operations, thus offering more accurate training for neural networks.

\section{CONCLUSION}

In this paper, we discussed problems that arise from "big data" and acknowledge the partial solution that data cooperatives provide. Next, we introduced a high level architecture for data cooperatives/unions that use anonymization and/or encryption to secure participants' data. We believe this new architecture we are developing will serve to complete the data cooperative model by adding privacy-protecting measures.

We outlined our approach to anonymization: edge swapping for vertices with lower cores and adding nodes/edges to preserve k-(core, property) at higher cores. Although our anonymization is in active development, we have no results to share at this time.

We used homomorphic encryption to ensure privacy where anonymization may degrade the accuracy of some metrics or where anonymization alone may not be sufficient to preserve the privacy of participants. Finally, we present results from our experiments showing the time complexity of several common graph operations on HE graphs: degree centrality, BFS, and farness centrality. These results show that use of homomorphic encryption is feasible for preserving privacy in data cooperatives.

The major limitation of our current work is that there is no efficient way to transform a ciphertext of one encryption scheme (HEAAN, BFV, etc.) into a ciphertext of a different encryption scheme other than decryption and re-encryption. While this is not a problem now, it will significantly impact our future work.

\section{FUTURE WORK}

While we have previously applied graph operations over HE graphs to locate super-spreaders, all of the graphs used were contact graphs. That is, each graph mapped vertices of a given type to vertices of that same type: people to people or location to location. Bridging different graphs would involve the use of transformation matrices (composed of attribute vectors) to map one graph's vertex set to another graph's vertex set. This is how we would accomplish linking graphs that contain different types of data. Consider the following example: 
Let $E$ be a transformation matrix mapping people (columns) to their current employers (rows). This matrix contains personal information and needs to be encrypted.

Let $R$ be a graph of road segments in a city with outbound edges to every road segment that can be reached from a given road segment. This graph is public data and does not need to be encrypted.

Let $T$ be the transformation matrix that maps people (columns) to the segments of road they use commuting to and from work. This information is personal and also needs to be encrypted.

Given a ciphertext vector of people, $p$, which can be obtained by selecting an employer in vector $e_{0}$ (single-member set), and performing $p=E^{T} e_{0}$, we can calculate $r$, the degree to which their commutes are centered on each segment of roadway $r=T p$. Likewise, an alternative query might be to take a road segment that is excessively congested during rush hour, and perform $p=\operatorname{BinMult}\left(T^{T}, r\right)$, which would result in $p$, the people who use that segment of road during their commute. From there, $p$ could be multiplied by $E$ to get $e_{1}$, the degree centrality of each employer relative to that congested road segment. With this result, a city could ask the most central employers in $e$ to stagger the start of their business day, reducing pollution caused by commuters sitting in traffic and the strain on the city's infrastructure.

With the mix of graphs and transformation matrices, a large number of meaningful aggregates can be composed through matrix operations. These transformation matrices have the added bonus of being meaningful attribute vectors. This means they can potentially be used to effectively train machine learning algorithms beyond their use in computing.

If we are to standardize these HE graph operations for analysis, there must be a well-defined algebra for transforming one type of graph data into another. We are working on adapting our HE graph algorithms to optimize for a hybrid approach where some data is public (plaintext) and other data, personal information, is encrypted. Our goal is to reduce all meaningful metrics to matrix operations.

\section{ACKNOWLEDGMENTS}

The authors would like to thank Noelle Mansilla for her help in organizing this paper.

\section{REFERENCES}

World Economic Forum, (2011, January). "Personal Data: The Emergence of a New Asset Class." [Online]. Available: http://www3.weforum.org/docs/ WEF_ITTC_PersonalDataNewAsset_Report_2011. pdf

D. Walsh, "How Credit Unions Could Help People Make the Most of Personal Data." 2019. [Online]. Available: https://mitsloan.mit.edu/ideas-made-tomatter/how-credit-unions-could-help-people-makemost-personal-data

J. Geuns and A. Brandusescu, "Shifting Power Through Data Governance." [Online]. Available: https://foundation.mozilla.org/en/data-futureslab/data-for-empowerment/shifting-power-throughdata-governance/

K. Ligett and K. Nissim, "Changes in Data Ownership and Usage." [Online]. Available: https://simons. berkeley.edu/sites/default/files/docs/11268/datacoopspresentationlongjanuary2019pptx.pdf

M. Dockendorf, R. Dantu, K. Morozov, and S. Bhowmick, "Locating Super-Spreaders with Homomorphically Encrypted Graphs," 2021.

J. Golbeck and J.L. Klavans, "Introduction to Social Media Investigation,” Waltham, MA: Syngress, 2015.

K. Liu and E. Terzi, "Towards identity anonymization on graphs," in Proceedings of the 2008 ACM SIGMOD International Conference on Management of Data. ACM, 2008.

P. Meyer, H. Siy, and S. Bhowmick, "Identifying Important Classes Of Large Software Systems Through KCore Decomposition," Advances in Complex Systems (ACS), vol. 17, 2014.

R. Laishram, A. E. Sariy"uce, T. Eliassi-Rad, A. Pinar, and S. Soundarajan, "Measuring and improving the core resilience of networks," in Proc. of WWW 2018 , 2018, pp. 609-618.

S. Sarkar, S. Bhowmick, and A. Mukherjee, "On rich clubs of path-based centralities in networks," in Proceedings of the 27th ACM International Conference on Information and Knowledge Management, ser. CIKM '18. New York, NY, USA: ACM, 2018, pp. 567-576. [Online]. https://dl.acm.org/doi/ $10.1145 / 3269206.3271763$.

V. Ufimtsev, S. Sarkar, A. Mukherjee, and S. Bhowmick, "Understanding stability of noisy networks through centrality measures and local connections," CIKM 2016, 2016. [Online]. Available: arXiv: 1609.05402

J. H. Cheon, K. Han, A. Kim, M. Kim, and Y. Song, "Bootstrapping for approximate homomorphic encryption," in EUROCRYPT 2018, ser. LNCS, vol. 10820, 2018, pp. 360-384.

A. Narayanan and V. Shmatikov, "De-anonymizing social networks," in 2009 30th IEEE Symposium on Security and Privacy, 2009, pp. 173-187.

H. Chen, I. Iliashenko and K. Laine, "When HEAAN meets FV: a new somewhat homomorphic encryption with reduced memory overhead," IACR ePrint Arch., vol. 2020/121, 2020 
R. Assam, M. Hassani, M. Brysch, and T. Seidl, “(k, d)-core anonymity: Structural anonymization of massive networks," in Proceedings of the 26th International Conference on Scientific and Statistical Database Management, ser. SSDBM 2014.

J. Cheng, A. W. Fu, and J. Liu, "K-isomorphism: Privacy preserving network publication against structural attacks," 2010

J. H. Cheon, A. Kim, M. Kim, and Y. S. Song, "Homomorphic encryption for arithmetic of approximate numbers," in ASIACRYPT 2017, ser. LNCS, vol. 10624, 2017, pp. 409-437.

A. Bulu c, J. T. Fineman, M. Frigo, J. R. Gilbert, and C. E. Leiserson, "Parallel sparse matrixvector and matrix-transpose-vector multiplication using compressed sparse blocks," in Proc. SPAA 2009. ACM, 2009, pp. 233-244.

X. Jiang, M. Kim, K. E. Lauter, and Y. Song, "Secure outsourced matrix computation and application to neural networks," in Proc. ACM CCS 2018. ACM, 2018, pp. 1209-1222.

X. Zhu and Z. Ghahramani, "Learning from labeled and unlabeled data with label propagation," 2003. [Online]. Available: http://mlg.eng.cam.ac.uk/zoubin/ papers/CMU-CALD-02-107.pdf

V. Mallawaarachchi, "Label propagation demystified." 2020. [Online]. Available: https://towardsdatascience. com/label-propagation-demystified-cd5390f 27472

L. Zhukov, "Structural analysis and visualization of networks course, hse." 2015. [Online]. Available: http://www.leonidzhukov.net/hse/2015/networks/ lectures/lecture17.pdf

S. B. Seidman, "Network structure and minimum degree," Social networks, vol. 5, no. 3, pp.269-287, 1983.

P. Holme, "Core-periphery organization of complex networks," Physical Review E, vol. 72, no. 4, p. 046111, 2005.

M. P. Rombach, M. A. Porter, J. H. Fowler, and P. J. Mucha, "Core-periphery structure in networks," SIAM Journal on Applied mathematics, vol. 74, no. 1, pp. 167-190, 2014.

V. Batagelj and M. Zaversnik, "An o(m) algorithm for cores decomposition of networks," CoRR, vol. cs.DS/0310049, 2003.

J. Cheng, Y. Ke, S. Chu, and M. T. Ozsu, "Efficient core decomposition in massive networks," in Proc. IEEE ICDE 2011. IEEE, 2011, pp. 51-62.

W. Khaouid, M. Barsky, V. Srinivasan, and A. Thomo, "Kcore decomposition of large networks on a single pc," Proceedings of the VLDB Endowment, vol. 9, no. 1, pp. 13-23, 2015.

A. Sala, X. Zhao, C. Wilson, H. Zheng, and B. Y. Zhao, "Sharing graphs using differentially private graph models," 2011

S. Ji, P. Mittal, and R. Beyah, "Graph data anonymization, de-anonymization attacks, and de-anonymizability quantification: A survey," IEEE Communications Surveys Tutorials, 2017.

S. Ji, W. Li, P. Mittal, X. Hu, and R. Beyah, "Secgraph: A uniform and open-source evaluation system for graph data anonymization and de-anonymization." USENIX Association, 2015.

M. Chase and S. Kamara, "Structured encryption and controlled disclosure," in Proc. ASIACRYPT 2010, ser. LNCS, vol. 6477. Springer, 2010, pp. 577-594.

X. Meng, S. Kamara, K. Nissim, and G. Kollios, "GRECS: graph encryption for approximate shortest distance queries," in Proc. 22nd ACM CCS Conference. ACM, 2015, pp. 504-517.

Q. Wang, K. Ren, M. Du, Q. Li, and A. Mohaisen, “Secgdb: Graph encryption for exact shortest distance queries with efficient updates," in Financial Cryptography and Data Security - 21st International Conference, FC 2017, ser. LNCS, vol. 10322. Springer, 2017, pp. 79-97.

C. Zhang, L. Zhu, C. Xu, K. Sharif, C. Zhang, and X. Liu, "Pgas: Privacy-preserving graph encryption for accurate constrained shortest distance queries," Information Sciences, vol. 506, pp. $325-345,2020$

S. Halevi, "Homomorphic encryption," in Tutorials on the Foundations of Cryptography. Springer International Publishing, 2017, pp. 219-276.

G. Zyskind, O. Nathan, A. Pentland, "Enigma: Decentralized Computation Platform with Guaranteed Privacy," 2015, [Online] Available: https://www.enigma. co/enigma_full.pdf.

L. Aslett, P. Esperanca, C. Holmes "A review of homomorphic encryption and software tools for encrypted statistical machine learning," 2015. [Online] Available: https://arxiv.org/pdf/1508.06574.pdf.

M. Newman, "Networks: An Introduction," USA: Oxford University Press, 2010.

A. López-Alt, E. Tromer, V. Vaikuntanathan. On-the-fly multiparty computation on the cloud via multikey fully homomorphic encryption. In Proceedings of the 44thannual ACM symposium on Theory of computing, pages 1219-1234. ACM, 2012.

A. Salau, R. Dantu, and K. Upadhyay "Mining and Chaining Fakeness, " 8th Annual Hot Topics in the Science of Security, Virtual Conference, Hosted by National Security Agency, April 13-15, 2021.

A. Salau, R. Dantu, "Data Cooperatives for Neighborhood Watch," IEEE International Conference on Blockchain and Cryptocurrency, Pages 1-8, Virtual Conference, May 3-6, 2021 